\title{
The effect of biofeedback training on intestinal function among patients with middle and low rectal cancer: a randomized controlled study
}

\author{
Li Liu ${ }^{1,2 \#}$, Xiaodan $\mathrm{Wu}^{1,2 \#}$, Qianwen Liu ${ }^{1,2}$, Caixing Tang ${ }^{1,2}$, Baojia Luo ${ }^{1,2}$, Yujing Fang ${ }^{1,2}$, Zhizhong Pan ${ }^{1,2}$, \\ Desen Wan $^{1,2}$, Meichun Zheng ${ }^{1,2}$ \\ ${ }^{1}$ Sun Yat-sen University Cancer Center, Guangzhou 510060, China; ${ }^{2}$ The Third Affiliated Hospital of Sun Yat-sen University, Guangzhou 510630, \\ China \\ Contributions: (I) Conception and design: L Liu, M Zheng, X Wu; (II) Administrative support: Z Pan, D Wan; (III) Provision of study materials or \\ patients: X Wu, Z Pan, M Zheng; (IV) Collection and assembly of data: L Liu, X Wu, Q Liu, B Luo; (V) Data analysis and interpretation: L Liu, C \\ Tang, Y Fang; (VI) Manuscript writing: All authors; (VII) Final approval of manuscript: All authors. \\ \#These authors contributed equally to this work. \\ Correspondence to: Meichun Zheng. Chief Superintendent Nurse, Head Nurse, Sun Yat-sen University Cancer Center, Guangzhou 510060 , China. \\ Email: zhengmch@sysucc.org.cn.
}

Background: To evaluate the effect of biofeedback on intestinal function among patients with middle and low rectal cancer.

Methods: Using a randomized controlled trial design, 109 patients with middle and low rectal cancer indicated to have preoperative radiochemotherapy, anterior resection of the rectum, and preventive stoma were randomly divided into three groups: the blank control group, the pelvic floor muscle exercise group, and the biofeedback training group. A 16-month intervention and longitudinal follow-up study was conducted, and a questionnaire on intestinal function by the Memorial Sloan-Kettering Cancer Center (MSKCC) was adopted into a Chinese version to evaluate patients' intestinal function situation.

Results: The intestinal function of the biofeedback training group was better than the blank control group and pelvic floor muscle exercise group. The total score of intestinal function and the scores of each dimension were statistically significant $(\mathrm{P}<0.05)$.

Conclusions: Biofeedback training could significantly improve the intestinal function of patients with middle and low rectal cancer, promote its recovery, and is thus worthy of clinical application.

Keywords: Biofeedback; rectal neoplasms; sphincter preservation operation (SPO); ileostomy; intestinal function

Submitted Aug 26, 2019. Accepted for publication Sep 06, 2019.

doi: 10.21037/atm.2019.09.62

View this article at: http://dx.doi.org/10.21037/atm.2019.09.62

\section{Introduction}

Rectal cancer is a common colorectal carcinoma causing death in China, for which middle and low rectal cancer accounts for $60-80 \%$ of the total incidence (1-3). With development of medical technology and people's higher requirements for the quality of life, many patients with rectal cancer of more than $2 \mathrm{~cm}$ between the neoplasm inferior margin and dentate line are able to have a sphincter preservation operation (SPO), so that the proportion of SPO takes up $62-85 \%$ in all rectal cancer operation $(4,5)$. Although bowel continuity can be realized through SPO, the inconvenience and mental stress of neostomy nursing throughout life should be avoided, as approximately $90 \%$ of patients face bowel symptoms such as an increase of defecation times, endless defecation, or even fecal incontinence, which are collectively referred to as anterior resection syndrome (ARS), and cause serious disturbance to daily life (6-9). The life quality for some 
patients with SPO is similar to that of patients with Mile's operation (5). At present, there is no specific therapy for ARS, and management is mainly based on experience and symptoms. Some researchers have reported the positive effect of drug use, anal plugging, rectal lavage, biofeedback training, and pelvic rehabilitation on rectal function $(3,10)$. However, most research has focused on treatment after the occurrence of postoperative symptoms and adoption of self pre- and post-control, while few studies have reported on the indications and timing of ARS prevention and ARS treatment (11). Biofeedback (BFT) is an emerging type of biological behavior therapy which centers on applying a biofeedback mechanism to collect the physiological activity information of patients. This information is processed and amplified by special equipment, and displayed by using familiar visual or auditory signals, so that patients can adjust physiological activities according to their own information drawn from the physiological activities observed; in this way, abnormal physiological change can be relieved or eliminated (12). BFT is considered to be an effective, safe, noninvasive, and economic method with little adverse effect (13). The present study is a prospective randomized controlled trial that explores the effect of biofeedback training on middle and low rectal cancer patients with SPO by dynamic analysis on patients' bowl function. The aim is to provide a practical basis for constructing reasonable rehabilitation training schemes of patients' anorectal function.

\section{Methods}

\section{Object of study}

This study is a prospective and randomized controlled trial. The selected patients had middle and low rectal cancer in a tumor hospital in Guangzhou from June 2015 to December 2016. The inclusion criteria were as follows: patients who were diagnosed with middle and low rectal cancer by pathology and pelvic MRI (with inferior margin being 2 to $8 \mathrm{~cm}$ to the dentate line) with expectation that the anus be preserved through surgery but with the patients needing to carry a temporary stoma; age of 18 to 75; with primary and higher education, and being able to communicate in Cantonese or Mandarin; being able to take care of themselves, and understanding and agreeing to participate in this study. The exclusion criteria were as follows: patients with cognitive or language disorders; metastatic rectal cancer or advanced neoplasms; with other malignant neoplasms being treated; subjected to pelvic or anus area surgery or ostomy in the past; combined with preoperative severe diseases such as hemorrhoids, anal fissure, anus fistula, rectocele, Crohn disease, irritable bowel syndrome, ulcerative colitis. Patients could be eliminated from the study for the following reasons: could not be treated according to the program, or with poor compliance; patients who asked to quit; patients had the following conditions after preoperative radiochemotherapy, and after re-assessment, the anus could not be preserved, radical resection could not be achieved and temporary neostomy was not needed, or temporary stoma could not be reversed; having postoperative intestinal obstruction, anastomotic fistula and other complications or not being able to eat through the mouth; the patients dying; the patients losing contact.

The study included 126 patients who met the criteria, and who were divided into the blank control group (Control Group 1), the pelvic floor muscle exercise group (Control Group 2), and the biofeedback training group (Intervention Group) (Figure 1). Patients were put into groups by being given a random number between 0 and 135 by a computer; the patients with numbers between 1 and 45 came under the blank control group, and totaled 42 cases; the patients with numbers between 46 and 90 came under the pelvic floor muscle exercise group, and totaled 40 cases; the patients with numbers between 91 to 135 came under the biofeedback training group, and totaled 44 cases. At the end of the study, 17 cases were missing, 2 of which were evaluated after preoperative radiochemotherapy to receive Mile's surgery that could not preserve the anus; 2 cases of radiochemotherapy were ineffective, neoplasms evolved, and they were unable to receive radical surgery; 3 cases were subject to SPO but no temporary stoma were required; 2 cases had anastomotic fistula after operation; 1 case had temporary stoma which could not be returned; 3 cases were reluctant to fill out questionnaires midway; 2 cases were of poor compliance and they were unwilling to perform functional exercises at home; and 2 cases were not contacted at a later stage. Finally we completed the study with 109 cases. Among them, 38 cases were in the blank control group, 36 cases were in the pelvic floor muscle exercise group, and 35 cases were in the biofeedback training group. The general information and the information on the treatment of diseases of each group are shown in Table 1; the three groups have comparability. 


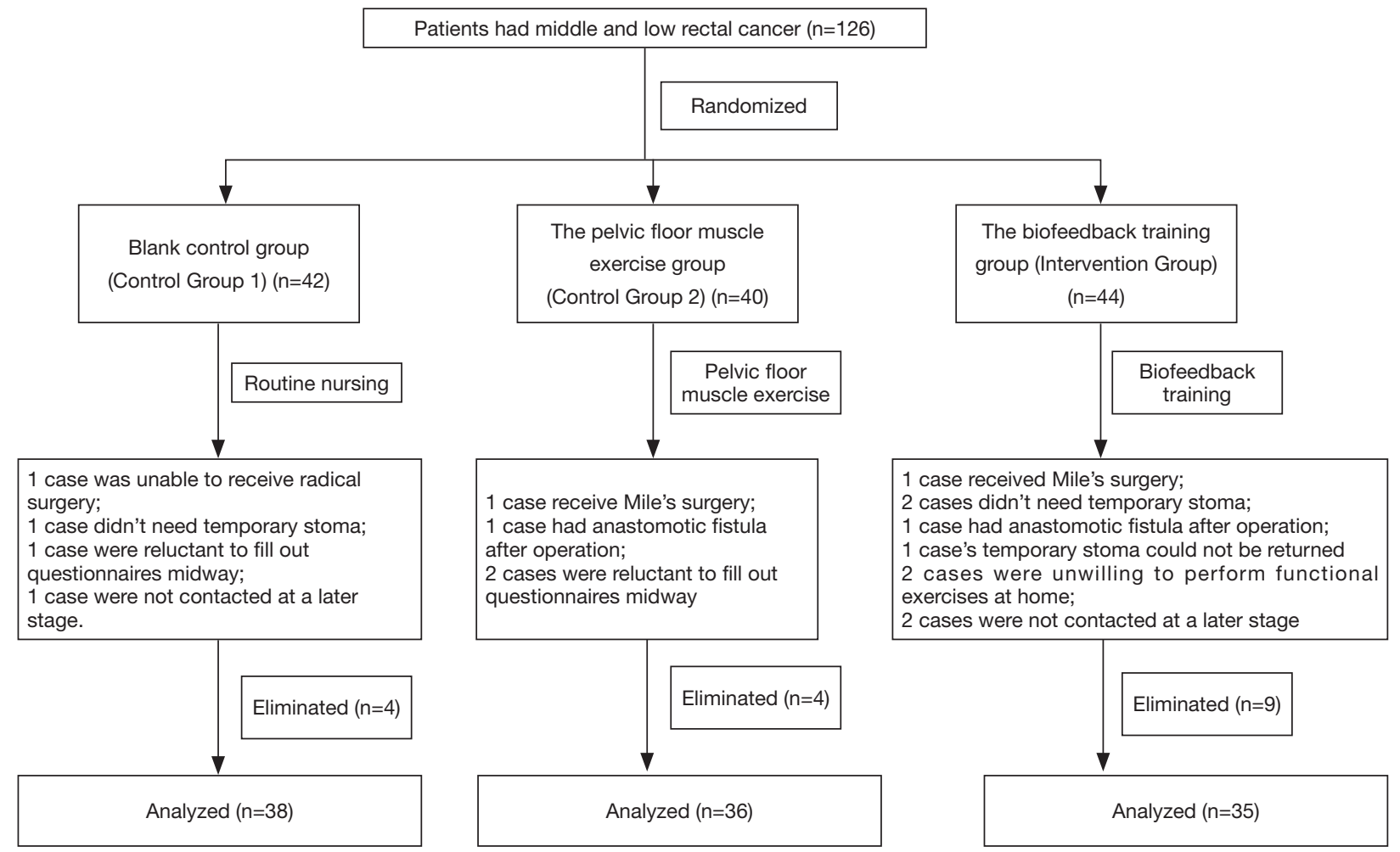

Figure 1 Flow chart of the design.

\section{Methods of intervention}

All three groups of patients received routine diagnosis and treatment. From the time when patients were diagnosed with rectal cancer, the inferior margin of the tumor was 2 to $8 \mathrm{~cm}$ from the dentate line; after examination by the attending doctor or multidisciplinary discussion, the treatment plan was determined to be preoperative radiochemotherapy with rectal neoplasm anterior resection (SPO) and the need to retain temporary stoma. The patients were in the group, 1 year after radical surgery, for a total of about 16 months. The methods of intervention for each group are detailed below.

The blank control group was given routine nursing. Radiochemotherapy and preoperative standard nursing were conducted, and diet, defecation posture and so on were guided.

The pelvic floor muscle exercise control group was given pelvic floor muscle functional exercise guidance on the basis of routine nursing.

The intervention group was given biofeedback training on the basis of routine nursing, with full-time nurses using high-resolution gastrointestinal dynamics test equipment to carry out biofeedback training for patients.

\section{Pelvic floor muscle function exercise}

Patients in the group were guided by the full-time nurses to conduct pelvic floor muscle exercise. The content and steps were as follows. First, patients and family members were informed of the purpose and method of the pelvic floor muscle exercise. Second, patients were guided to conduct pelvic floor muscle exercise; patients could choose the position of lying on their back or being in standing position or sitting position according to their own situation; to tighten the pelvic floor muscles (for holding in feces), patients were encouraged to tighten and lift muscles as much as possible for 5 to $10 \mathrm{~s}$, and then to relax and rest for $10 \mathrm{~s}$; this was considered to be 1 repetition. This workout could be done in 5 sessions a day, 10 repetitions per session. It was carried out every day before the operation. After the operation, the exercise was suspended for 1 week, and resumed after physical recovery, which lasted for a total of about 16 months. Third, a video was released of the exercise method guidelines and pelvic floor muscle movement as a 
Table 1 Comparison of general data from the three groups of patients $(n=109)$

\begin{tabular}{|c|c|c|c|c|c|}
\hline Item & $\begin{array}{l}\text { Blank control } \\
\text { group }(n=38)\end{array}$ & $\begin{array}{l}\text { Pelvic floor muscle } \\
\text { exercise group } \\
\quad(n=36)\end{array}$ & $\begin{array}{l}\text { Biofeedback } \\
\text { training group } \\
\quad(n=35)\end{array}$ & Statistical value & $P$ value \\
\hline Gender & & & & 0.554 & 0.758 \\
\hline Male & 30 & 27 & 25 & & \\
\hline Educational level & & & & 3.021 & 0.933 \\
\hline Primary school & 10 & 11 & 9 & & \\
\hline Junior high school & 11 & 15 & 12 & & \\
\hline Senior high school/polytechnic school & 10 & 6 & 9 & & \\
\hline Distance from tumor to anus $(\mathrm{cm})$ & $5.11 \pm 1.87$ & $5.06 \pm 1.88$ & $5.00 \pm 1.63$ & 0.031 & 0.969 \\
\hline Tumor clinical stage & & & & 1.119 & 0.572 \\
\hline II & 6 & 8 & 9 & & \\
\hline III & 32 & 28 & 26 & & \\
\hline Surgery method & & & & 0.165 & 0.921 \\
\hline Celiotomy Dixon & 5 & 4 & 5 & & \\
\hline Laparoscopically assisted Dixon & 33 & 32 & 30 & & \\
\hline
\end{tabular}

demonstration to patients for their self-exercise use after returning home. Fourth, patients were assessed on whether they correctly grasped the exercise methods. On the second to the third day after training, the patients were told to lie on their back. Full-time nurses wearing gloves with the index finger coated with paraffin oil gently inserted the finger into the patients' anus and asked the patients to conduct the pelvic floor muscle exercise; it was correct to feel tension in the anus tube with the finger. Fifth, at least 1 call a week was made after discharge, urging patients to stick with the exercise.

\section{Biofeedback training}

When using the InSIGHT PHNS-A biofeedback training and treatment system, i.e., Sandhill's solid anorectal high resolution pressure measuring device, the pasted threechannel body surface electrode of the abdominal oblique muscle and the vertical inserted anal tube electrode were placed on the body surface and the lower rectum in the anus respectively to form the current circuit. Then, the biofeedback device was connected to observe the EMG waveform and dynamic change process of the pressure curve of the patients in the process of anal contraction on the display screen of the system computer, while making the pressure curve as close as possible to normal contraction pressure curve. The full-time nurses chose the appropriate biofeedback course for the patients according to the change of EMG, trained and guided the patients to identify their abnormal and normal EMG signals, and guided the repeated anal shrinkage, relaxation, defecation and other actions, and then repeated the self-training. This was repeated 3 times a week and 20 minutes per session; 4 consecutive weeks were considered 1 course of treatment, and a total of 4 courses were conducted. The 1 st course of treatment was the 1 st week to 4 th week after being included in the group. The 2 nd course of treatment was the 1 st week to 4th week after 1 month of accepting radical surgery. The 3 rd course of treatment was the 1st week to 4th week after accepting stoma returning operation. The 4th course of treatment was the 1st week to 4 th week after 3 months of 
the stoma returning operation. The full-time nurses also guided the patients to carry out the pelvic floor muscle exercise method, and required the patients to adhere to the pelvic floor muscle function exercise at home in the interval of biofeedback training, with the same method as that of the pelvic floor muscle functional exercise group.

\section{Evaluation indicators and methods}

The intestinal function of patients was evaluated before intervention (T1) 1 day before radical surgery (T2), 4 days after stoma returning operation (T3), 3 months after stoma returning operation (T4), and 9 months after stoma returning operation (T5); a total of 5 questionnaires were conducted by using the Chinese version of the Memorial Sloan-Kettering Cancer Center (MSKCC) intestinal function questionnaire, in which T1, T2, and T3 were completed in the ward, and T4 and T5 were completed by the patients in the clinic upon appointment. If the patient met any of the termination criteria, such a patient was withdrawn from the research. When the patient was included in the group, the purpose and content of the study were explained to the patient in the hospital proctology ward, the consent of the patient and the family member was obtained, the informed consent was completed, and the questionnaire was issued to the patient. The researchers used the unified instructions to explain the completion method of the questionnaire; in the process of completing the questionnaire, the unclear questions were explained at any time, and the interpretation of each entry was consistent without being guiding. Some of the disease-related data were obtained by inquiring about medical record data uniformly, and the rest of the information was completed by the patients. For those who had difficulty in completing the questionnaire independently, the researchers read the questionnaire for them, the patients answered it, and the researchers filled it out on their behalf, truthfully according to the patients' opinions. The questionnaire was distributed and collected uniformly by the researchers themselves. The researchers contacted the patients in advance for follow-up, and made an appointment with the patients for the time to fill out the questionnaire.

The Chinese version of the Memorial Sloan-Kettering Cancer Center (MSKCC) intestinal function questionnaire is a patient self-assessment questionnaire developed by Temple et al. (14) in 2005. The Chinese version of the questionnaire included 4 factors of defecation frequency, defecation urgency, defecation affected by diet, and abnormal defecation feeling, a total of 18 entries. The 1st question is "For how many days during the last 4 weeks did you defecate normally?" According to the range of daily defecation times of the population studied, scoring for each item is divided into 5 equal parts, and each is assigned with a value between 1 and 5 points according to the degree of defecation from more to less; the rest of the entries adopt Likert 5-level grading which includes "always", "often", "sometimes", "rarely", and "never"; these are assigned a value of 1 to 5 points respectively, in which the 4th, 5th, 7 th, 11th and 12th entries adopt reverse grading. The sum of scores of all entries was the total score of the scale, and the higher the total score, the better the intestinal function of the patients. In this study, the Chinese version translated by Hou et al. (15) was used for investigation; the Cronbach's $\alpha$ coefficient of the total score of the Chinese version of the questionnaire for each factor was 0.602 to 0.856 , the reliability of the retest was 0.662 to 0.893 , and the questionnaire had good convergent validity and known group validity (15).

\section{Statistical methods}

After data collection, the data were checked and input by two people using SPSS 15.0 software and described using frequency with mean \pm standard deviation. The equilibrium test between the two sets of data was carried out by paired $t$-test and Chi-square test; the change trend of the intestinal function scale score at different time points was analyzed by using RMANOVA of a general linear model. $\mathrm{P}<0.05$ indicates that the difference is statistically significant.

\section{Results}

\section{Comparison of general data from the three groups of patients}

Age, gender, education level, tumor location and stage, and surgery methods of the three groups of patients had no statistical differences, and these data were comparable (see Table 1).

\section{Comparison of the intestinal function status across the three groups of patients}

The total score of anorectal function and the scores of each dimension of the three groups were not statistically different before treatment $(\mathrm{F}=0.236-2.205, \mathrm{P}>0.05)$, and 
Table 2 Comparison of bowel function between the pelvic floor muscle exercise group and the biofeedback group

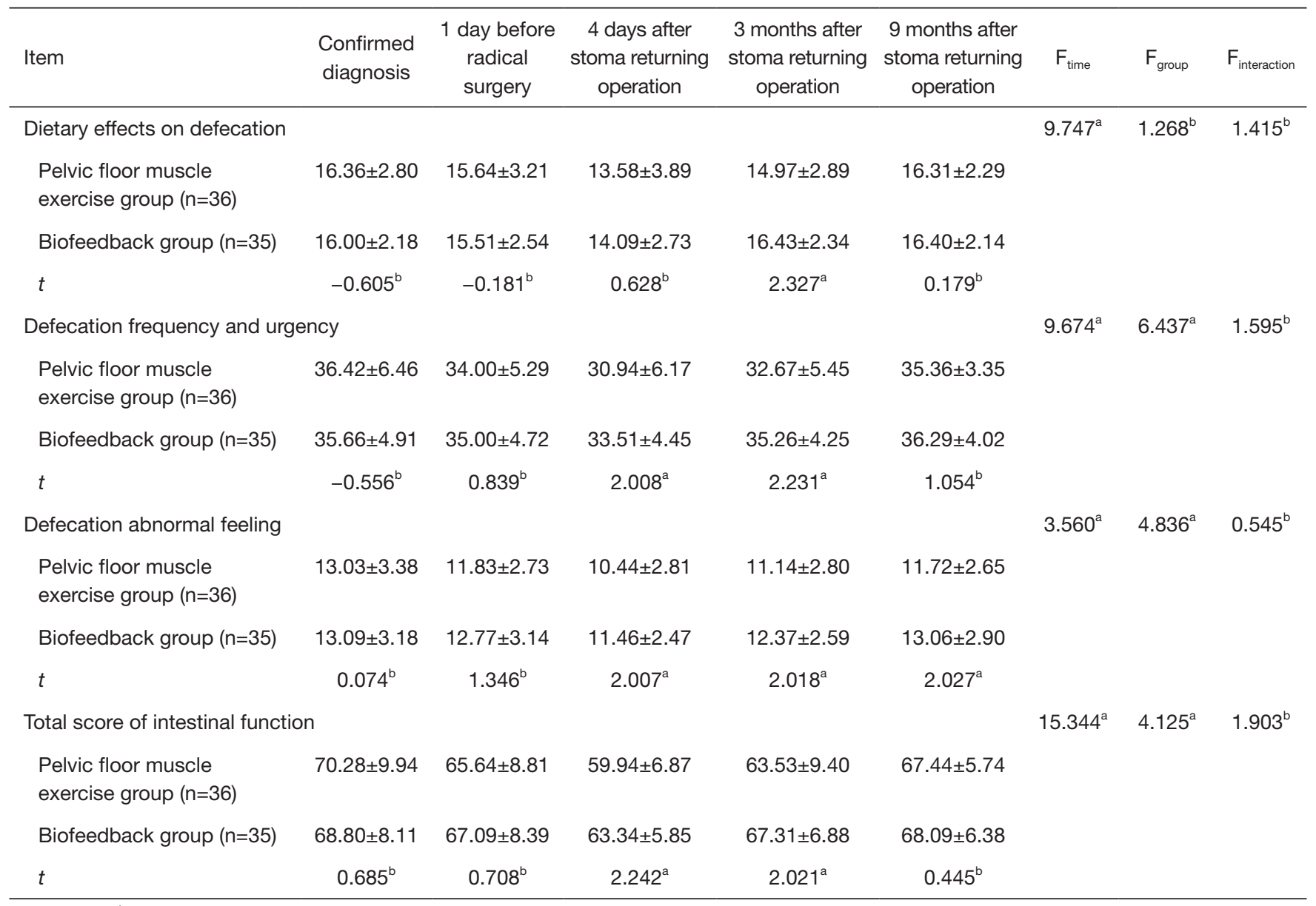

${ }^{a}, P<0.05 ;{ }^{b}, P>0.05$.

the three groups' data were comparable. The total score of the intestinal function scale and the scores of each dimension had significant changes over time $(\mathrm{P}<0.05)$. The score was the highest at the time of diagnosis, decreased after preoperative radiochemotherapy, bottoming out after 1 month of radical surgery, and gradually recovering to be close to the preoperative level with time. Comparing the biofeedback training group with the blank control group, the differences of change between neighboring times of total score of intestinal function and scores of each dimension in both group's patients were all statistically significant $(\mathrm{P}<0.05)$. Comparing the biofeedback training group with the pelvic floor muscle exercise group, the differences of change between neighboring times of total score of intestinal function and the scores of the dimensions of defecation frequency, defecation urgency and defecation abnormal feeling in both groups' patients were all statistically significant $(\mathrm{P}<0.05)$. See Tables 2 and 3 for details.

\section{Discussion}

\section{Biofeedback training can significantly improve intestinal function of patients with low and middle rectal cancer}

This study showed that the total score of intestinal function and scores of each dimension of the three groups' patients had significant changes over time, which indicated that the patients' intestinal function will change to varying 
Table 3 Comparison of bowel function between the blank control group and the biofeedback group

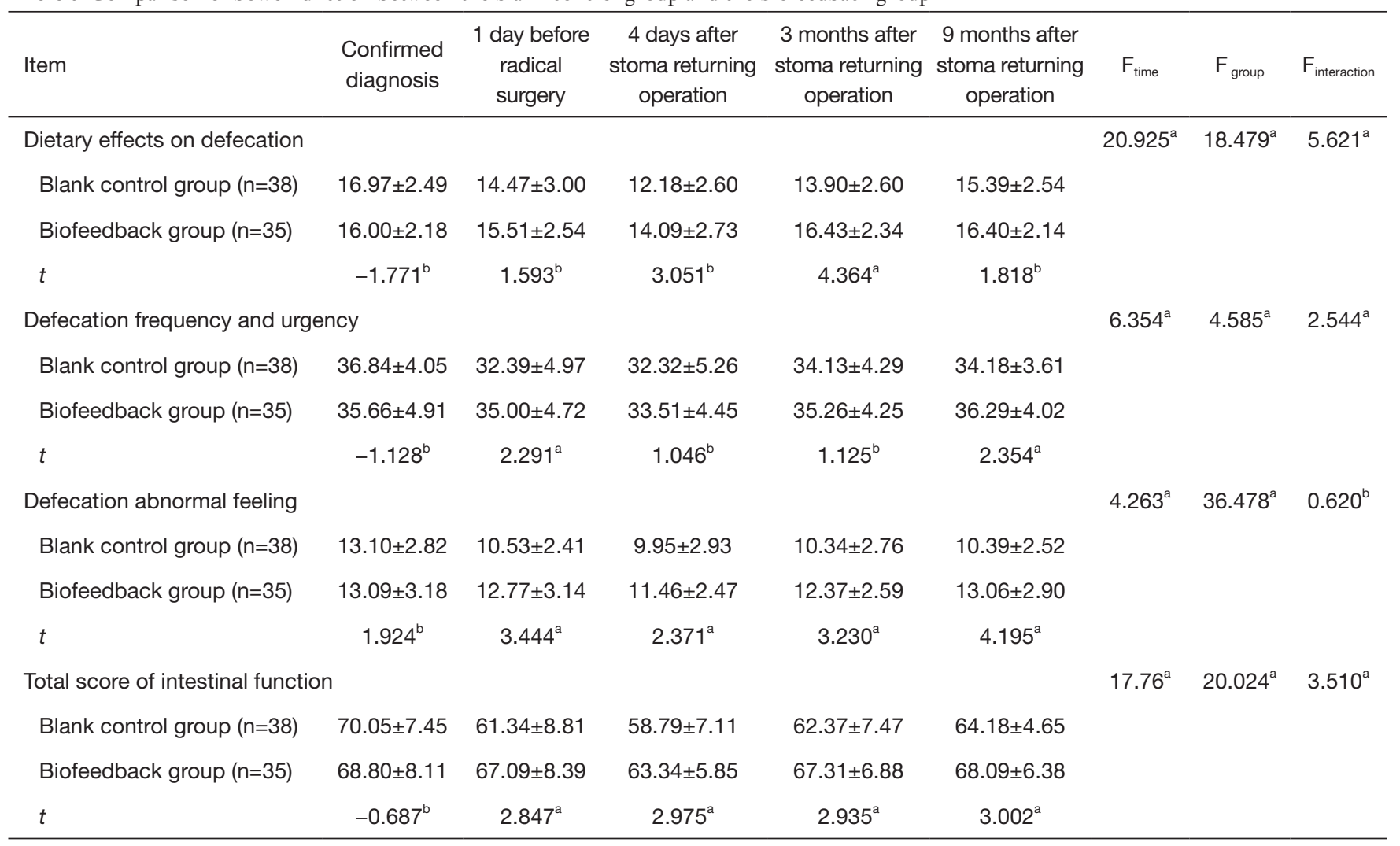

${ }^{\mathrm{a}}, \mathrm{P}<0.05 ;{ }^{\mathrm{b}}, \mathrm{P}>0.05$.

degrees even without any intervention after treatment. After receiving radiochemotherapy, the scores of intestinal function of patients were reduced. After receiving the radical surgery and stoma returning operation, the scores of intestinal function of patients were further reduced, and then the intestinal function gradually recovered with the passage of time. It shows that radiochemotherapy and operation had significant effects on the intestinal function of patients, which is consistent with previous studies $(16,17)$. Changes in the intestinal function of the patients in the biofeedback training group indicate that biofeedback training cannot completely prevent physiological and functional changes in the anorectum.

In the biofeedback training group, the total score of intestinal function and scores of each dimension were significantly higher than those of the blank control group during the post-intervention change, which suggested that biofeedback training can significantly improve intestinal dysfunction caused by treatment on patients with middle and low rectal cancer. That is, biofeedback training enables patients to recover faster, earlier, and closer to the pretreatment state at the end of the measurement. This is because the SPO of the middle and low rectal cancer often needs to remove part of the rectum close to the dentate line (5), causing damage to the rectal stool receptor, resulting in a smaller rectal volume and a paresthesia. Previous scholars (10) also pointed out that defecation dysfunction was more common than defecation incontinence in patients with middle and low rectal cancer, and its postoperative effects were more common and more severe $(3,18,19)$. In addition, visceral afferent neurological dysfunction and fibrosis caused by radiochemotherapy led to residual rectal sensitivity disorder which led to abnormal intestinal function of patients. Biofeedback training can adjust the central autonomic pathway through visual and auditory information feedback (20), and can also change the local nerves and body fluids in the hypothalamus and cerebral cortex to adjust the neural reflex $(12,20)$ to improve 
the sensory function of the rectum, thereby improving the intestinal function and mental state of the patients.

The results of this study show that, in the biofeedback training group, the total score of intestinal function and scores of the dimensions of defecation frequency, urgency and abnormality after intervention were significantly higher than those of the pelvic floor muscle exercise group. Previous studies have shown that pelvic floor muscle exercise can improve anorectum function in patients with middle and low rectal cancer by strengthening muscle contraction training (11). However, since the pelvic floor muscle exercise was only done at patients' homes, there was no objective way to evaluate whether the patients really master the exercise method, and it was difficult to determine whether the exercise was effective. Therefore, the medical staff did not supervise whether the exercise was performed correctly, and the patients may have had inherent inefficiency or improper implementation, which might have affected the exercise effect (11). In contrast, through biofeedback training, patients can visually and intuitively experience information about pelvic floor muscle activity and can also provide information on the improvement of pelvic muscle strength to medical personnel for monitoring, which can ensure the correctness and effectiveness of training. This study also suggested that biofeedback training was greater than the pelvic floor muscle exercise group to the patients. Moreover, biofeedback training is non-invasive, inexpensive, and has fewer side effects. It is an ideal method for patients with middle and low rectal cancer and is worthy of promotion.

\section{Measures to improve the biofeedback training intervention}

Biofeedback training is safe, effective, inexpensive, and has few side effects, but it takes a while to produce the desired result. The patients' training compliance is critical to the training effect. Previous studies have also confirmed $(12,13)$ that the efficacy of biofeedback training is not related to the patients' age, gender, and course of the disease, but related to whether the patients can adhere to treatment and the course of treatment. Before the start of the study, the intervention program was revised and improved several times in order to ensure the patients' convenience as much as possible under the premise of ensuring the effect to improve the patients' compliance. In the process of intervention, the researchers also fully understood the psychological and social environment of each patient and took targeted measures to improve patients' compliance. For example, the working time of the nurse who was responsible for the biofeedback training was flexibly arranged, and the biofeedback training was performed according to the time of the patients on the weekend. In order to improve the home training compliance of some patients who tended to forget the training time, the researchers regularly sent information, made special calendars to remind them of regular training, encouraged their families to participate, and urged patients to return to hospital for treatment on time.

\section{Conclusions}

In summary, biofeedback training cannot completely avoid the occurrence of intestinal dysfunction in patients with middle and low rectal cancer, but it can reduce the degree of intestinal dysfunction and promote the recovery of intestinal function of patients with rectal cancer during the course of treatment. All the patients in this study were all middle and low rectal cancer patients who received radiochemotherapy, surgery, and temporary stoma. The sample types were relatively simple and were high-risk patients with ARS, which can represent the situation of these patients better. These patients received biofeedback training immediately after they were included in the groups, which changed the method of training in the past (training was only conducted if there was a problem) and played a preventive role, so that it allowed patients to recover their functions and benefit earlier. The tracking time was relatively long, and the total time length was 16 months (9 months after the stoma returning operation). The patients were already in a stable state during the recovery period, and the researchers could comprehensively evaluate the effect of the intervention. The deficiencies in this investigation were that the number of patients in each group of this study was low, and it was a single-center study; thus, the representativeness needs to be strengthened. Large-sample and multi-center research can be carried out in the future.

\section{Acknowledgments}

Funding: Scientific research project: this work was supported by the grants from the National Natural Science Foundation of China (No. 81871991, No. 81502459, and No. 81772595), the Guangzhou Science and Technology Planning Projects (Health Medical Collaborative Innovation 
Program of Guangzhou) (grant No. 201803040019 and No. 201400000001-4), and the Guangdong Science and Technology Planning Project in 2015 (2014A020212105).

\section{Footnote}

Conflicts of Interest: The authors have no conflicts of interest to declare.

Ethical Statement: The authors are accountable for all aspects of the work in ensuring that questions related to the accuracy or integrity of any part of the work are appropriately investigated and resolved. The study has obtained ethics approval from Ethics Committee of Sun Yat-sen University Cancer Center, the number of the approval was 5010-2014-04-01. Written informed consent was obtained from the patient for publication of this manuscript and any accompanying images.

\section{References}

1. Sun KX, Zheng RS, Zhang SW, et al. Report of Cancer Incidence and Mortality in Different Areas of China, 2015. China Cancer 2019;28:1-11.

2. Torre LA, Bray F, Siegel RL, et al. Global cancer statistics, 2012. CA Cancer J Clin 2015;65:87-108.

3. Kim YA, Lee GJ, Park SW, et al. Multivariate Analysis of Risk Factors Associated With the Nonreversal Ileostomy Following Sphincter-Preserving Surgery for Rectal Cancer. Ann Coloproctol 2015;31:98-102.

4. Du D, Su Z, Wang D, et al. Optimal Interval to Surgery After Neoadjuvant Chemoradiotherapy in Rectal Cancer: A Systematic Review and Meta-analysis. Clin Colorectal Cancer 2018;17:13-24.

5. Dulskas A, Miliauskas $P$, Tikuisis R, et al. The functional results of radical rectal cancer surgery: review of the literature. Acta Chir Belg 2016;116:1-10.

6. Zhang MF, Zheng MC, Liu WY, et al. The influence of demographics, psychological factors and self-efficacy on symptom distress in colorectal cancer patients undergoing post-surgical adjuvant chemotherapy. Eur J Oncol Nurs 2015;19:89-96.

7. Mahalingam S, Seshadri RA, Veeraiah S. Long-Term Functional and Oncological Outcomes Following Intersphincteric Resection for Low Rectal Cancers. Indian J Surg Oncol 2017;8:457-61.

8. Bryant CL, Lunniss PJ, Knowles CH, et al. Anterior resection syndrome. Lancet Oncol 2012;13:e403-8.

9. Wu XD, Qin HY, Zhang JE, et al. The prevalence and correlates of symptom distress and quality of life in Chinese oesophageal cancer patients undergoing chemotherapy after radical oesophagectomy. Eur J Oncol Nurs 2015;19:502-8.

10. Chen TY, Emmertsen KJ, Laurberg S. Bowel dysfunction after rectal cancer treatment: a study comparing the specialist's versus patient's perspective. BMJ Open 2014;4:e003374.

11. Kye BH, Kim HJ, Kim G, et al. The Effect of Biofeedback Therapy on Anorectal Function After the Reversal of Temporary Stoma When Administered During the Temporary Stoma Period in Rectal Cancer Patients With Sphincter-Saving Surgery: The Interim Report of a Prospective Randomized Controlled Trial. Medicine (Baltimore) 2016;95:e3611.

12. Skardoon GR, Khera AJ, Emmanuel AV, et al. Review article: dyssynergic defaecation and biofeedback therapy in the pathophysiology and management of functional constipation. Aliment Pharmacol Ther 2017;46:410-23.

13. Heymen S, Jones KR, Scarlett Y, et al. Biofeedback treatment of constipation: a critical review. Dis Colon Rectum 2003;46:1208-17.

14. Temple LK, Bacik J, Savatta SG, et al. The development of a validated instrument to evaluate intestinal function after sphincter-preserving surgery for rectal cancer. Dis Colon Rectum 2005;48:1353-65.

15. Hou XT, Pang D, Lu Q, et al. Validation of the Chinese version of the low anterior resection syndrome score for measuring bowel dysfunction after sphincter-preserving surgery among rectal cancer patients. Eur J Oncol Nurs 2015;19:495-501.

16. Hou XT, Pang D, Lu Q, et al. Bowel Dysfunction and Self-management for Bowel Symptoms After Sphincter-Preserving Surgery: A Cross-sectional Survey of Chinese Rectal Cancer Patients. Cancer Nurs 2017;40:E9-16.

17. Landers M, McCarthy G, Savage E. Bowel symptom experiences and management following sphincter saving surgery for rectal cancer: A qualitative perspective.Eur J Oncol Nurs 2012;16:293-300.

18. Wang YH, Zhang Y, Gong JQ, et al. Application of the transtheoretical model and stages of change to patients receiving training after anus-preserving operation. Journal of Nursing Science 2012,27:30-2.

19. Mehrvarz S, Towliat SM, Mohebbi HA, et al. Comparison 
Page 10 of 10

of Colonic J-pouch and Straight Coloanal anastomosis after Low Anterior Resection. Iran Red Crescent Med J 2013;15:32-5.

20. Emmanuel AV, Kamm MA. Response to a behavioural

Cite this article as: Liu L, Wu X, Liu Q, Tang C, Luo B, Fang Y, Pan Z, Wan D, Zheng M. The effect of biofeedback training on intestinal function among patients with middle and low rectal cancer: a randomized controlled study. Ann Transl Med 2019;7(21):605. doi: 10.21037/atm.2019.09.62
Liu et al. Biofeedback training on intestinal function

treatment, biofeedback, in constipated patients is associated with improved gut transit and autonomic innervation. Gut 2001;49:214-9. 